\title{
77. PRELIMINARY REPORT ON BENTHONIC FORAMINIFERA FROM THE MID-ATLANTIC RIDGE: LEG 37 DSDP
}

\author{
K. Hooper and P. Jones, Department of Geology, Carleton University, Ottawa, Canada
}

\section{INTRODUCTION}

Benthonic foraminifera were studied from 46 sediment samples of five Leg 37 drill holes (Table 1). The samples range in age from late Miocene to Pleistocene. The aims of the study were to record the species present and to attempt an evaluation of the paleoenvironments that they represent.

The chief difficulties encountered in preparing this report were the short time available (effectively less than 4 months), the page limitation, and the scattered nature of the literature. Because of these factors the aims of the study were not completely achieved, and therefore an expanded treatment of the data is planned for a later date.

\section{RESULTS}

1. All of the samples studied are deep-sea oozes containing abundant foraminifera.

2. Although no silt or sand layers on graded beds were seen, some downslope displacement is considered likely because many foraminifera were damaged. However, biological rather than physical agents could be responsible.

3. Commonly the benthonic foraminifers are small in size.

4. Benthonic foraminifers account for less than $1 \%$ of the foraminiferal fauna in each sample.

5. Although the number of individual benthonic specimens is low, the number of species is high.

6. Many of the species are extant and their presentday preferred habitats are at least partially known. Unfortunately, the habitat data available from the literature vary greatly in quantity and quality from species to species and from author to author. In particular, depth ranges of species are variable. Nevertheless an attempt here is made to classify the species into habitat groups as follows:

Group I: Tropical, warm, shallow. Littoral.

Group II: Tropical, warm, shallow. Inner neritic $(0-30 \mathrm{~m})$.

Group III: Tropical, warm to cool. Neritic (30$100 \mathrm{~m})$.

Group IV: Temperate to polar, cool to cold. Neritic $(0-100 \mathrm{~m})$.

Group V: All climatic zones, cool to cold. Deep (below $100 \mathrm{~m}$ ).

The boundaries between groups are only intended to be approximate.

7. The faunas listed in Appendix I comprise the species that are identified with a high degree of con-
TABLE 1

Sample Data

\begin{tabular}{|c|c|c|}
\hline $\begin{array}{l}\text { Sample } \\
\text { No. }\end{array}$ & $\begin{array}{l}\text { DSDP Sample } \\
\text { (Interval in } \mathrm{cm} \text { ) }\end{array}$ & Age \\
\hline 1 & $332 \mathrm{~A}-4-4,60-62$ & NN15?, N20? late Pliocene \\
\hline 3 & $332 \mathrm{~A}-5-3,128-130$ & NN15?N20? late Pliocene \\
\hline 5 & $332 \mathrm{~A}-5-6,8-10$ & NN15, N20, late Pliocene \\
\hline 7 & $332 \mathrm{~A}-6-2,42-44$ & NN15, N20, late Pliocene \\
\hline 8 & $332 \mathrm{~B}-1-1,16-18$ & NN16, N20/N21, late Pliocene \\
\hline 9 & $332 \mathrm{~B}-1-2,11-13$ & NN15, N20/N21, late Pliocene \\
\hline 11 & $332 \mathrm{~B}-1-4,10-12$ & NN15, N20/N21, late Pliocene \\
\hline 12 & $333 \mathrm{~A}-1-1,86-88$ & NN15, N19, late Pliocene \\
\hline 13 & $333-2-1,86-88$ & NN16, N21, late Pliocene \\
\hline 15 & $333-2-3,86-88$ & NN16, N21, late Pliocene \\
\hline 17 & $333-2-5,25-26$ & NN16, N21, late Pliocene \\
\hline 19 & $333-3-1,86-88$ & NN15?, N20, late Pliocene \\
\hline 21 & $333-3-3,86-88$ & NN15?, N20, late Pliocene \\
\hline 23 & $333-3-5,86-88$ & NN15?, N20, late Pliocene \\
\hline 25 & $333-4-1,86-88$ & NN15, N20, late Pliocene \\
\hline 27 & $333-4-3,86-88$ & NN15, N20, late Pliocene \\
\hline 29 & $333-4-5,86-88$ & NN15, N20, late Pliocene \\
\hline 30 & $333-5-2,86-88$ & NN15, N19, late Pliocene \\
\hline 32 & $333-6-1,86-88$ & NN15, N19, late Pliocene \\
\hline 34 & $333-7-2,86-88$ & NN15, N19, late Pliocene \\
\hline 35 & $334-1-1,86-88$ & NN21, N23, Pleistocene \\
\hline 37 & $334-2-1,86-88$ & NN11, N17, late Miocene \\
\hline 39 & $334-2-3,86-88$ & NN11, N17, late Miocene \\
\hline 40 & $334-2-4,86-88$ & NN11, N17, late Miocene \\
\hline 42 & $334-2-6,86-88$ & NN11, N17, late Miocene \\
\hline 44 & $334-4-1,86-88$ & NN11, N17, late Miocene \\
\hline 45 & $334-5-3,86-88$ & NN11, N17, late Miocene \\
\hline 47 & $334-6-1,86-88$ & NN11, N17, late Miocene \\
\hline 49 & $334-7-1,86-88$ & NN11, N16, late Miocene \\
\hline 51 & $334-7-3,114-116$ & NN11, N16, late Miocene \\
\hline 53 & $334-7-5,86-88$ & NN11, N16, late Miocene \\
\hline 55 & $334-8-1,43-45$ & NN11, N16, late Miocene \\
\hline 57 & $334-9-2,103-105$ & NN11, N16, late Miocene \\
\hline 59 & $334-9-4,88-90$ & NN11, N16, late Miocene \\
\hline 61 & $334-11-3,86-88$ & NN10, N16, late Miocene \\
\hline 63 & $334-12-2,86-88$ & NN10, N16, late Miocene \\
\hline 65 & $334-12-4,91-93$ & NN10, N16, late Miocene \\
\hline 67 & $334-13-4,96-98$ & NN10, N15, late Miocene \\
\hline 68 & $334-13-5,86-88$ & NN10, N15, late Miocene \\
\hline 70 & $334-14-1,84-86$ & $\mathrm{NN} 10, \mathrm{~N} 15$, late Miocene \\
\hline 71 & $335-1-2,86-88$ & NN18, N21, late Pliocene \\
\hline 73 & $335-1-4,86-88$ & NN18, N21, late Pliocene \\
\hline 75 & $335-2-3,86-88$ & NN15, N20, late Pliocene \\
\hline 77 & $335-2-5,86-88$ & NN15, N19, early Pliocene \\
\hline 79 & $335-4-2,86-88$ & NN11, N16/17 late Miocene \\
\hline 80 & $335-4-3,86-88$ & NN11, N16/17 late Miocene \\
\hline
\end{tabular}

Note: Ages based on Leg 37 Site Reports (Chapters 2-5, this volume).

fidence and whose present-day habitats are judged to be moderately well known. The arrangement is sequential by group. Species that only seem to be known as fossils and others that have not yet been positively identified are not given in this report. 
8. A few species are present that are known to live in shallow water attached to seaweed (i.e., Cibicides lobatulus). Others are present that have the concaveconvex shape that often is associated with this mode of life. Such forms could have drifted into the MidAtlantic area on the seaweed and were subsequently deposited in the sediments.

9. Species that are reported in the literature to be confined to bathyal and abyssal depths in the modern oceans are found in each and every sample.

\section{CONCLUSIONS}

1. There are no tropical-littoral group I species.

2. The two species that fall into the tropical-inner neritic group II also range into other (deeper) groups and consequently are eurybathic. Group II stenothermal and stenobathic species are absent.

3. Excepting only Fissurina crebra, species placed in group III also occur in groups IV and/or V, and are probably eurythermal and/or eurybathic.

4. Group IV species also occur in group V. Modern species known to live in the group IV habitat are adapted to cool and cold waters. Similar temperature conditions prevail in the deep waters of the group V habitat. This may account for the co-occurrence.

5 . More than $98 \%$ of the species occur in deep water group V, and approximately $50 \%$ of the species are confined to group $\mathrm{V}$.

6. Some bathyal and abyssal restricted species (group V) occur in each of the 46 samples investigated.

7. Bathyal to abyssal bottom conditions (possibly deeper than about $1000 \mathrm{~m}$ ) are indicated by the benthonic foraminiferal faunas during late Miocene to Pleistocene time in the area investigated. Precise depth determination of each sample is currently being attempted and will be reported later.

\section{APPENDIX I: FAUNA LIST}

Bolivina spathulata Williamson: II, III, V: Sample 32.

Uvigerina auberiana d'Orbigny: II, III, V: Samples 3, 7, 8, 9, 13, 15, $17,19,23,25,27,37,70$.

Fissurina crebra (Matthes): III: Samples 17, 37, 44.

Angulogerina angulosa (Williamson): III, IV, V: Samples 13, 15, 27, $42,44,45,47,51,55,59,65$.

Bolivina pusilla Schwager: III, V: Samples 9, 19, 21, 25, 39, 40, 42, 44 , $55,63$.

Bolivina robusta Brady f.A: III? IV? V?: Sample 44.

Bulimina alazanensis Cushman: III? IV? V: Samples 7, 9, 25, 29, 32, $37,39,40,42,47,49,51,55,57,59,61,63,67,73,75,77,80$.

Bulimina senta Finlay: III? IV? V: Samples 9, 17, 21, 39, 45, 47, 51, 55 $59,70,77$.

Cassidulina crassa d'Orbigny: III? IV, V: Sample 61.

Cassidulina subglobosa Brady: III, IV, V: Samples 1, 3, 5, 7, 8, 9, 11 , $12,13,15,17,19,21,23,25,27,29,30,32,34,35,37,39,40,42$. $44,45,47,49,51,53,55,57,59,61,63,65,67,68,70,71,73,75$, $77,79,80$.

Cassidulinoides bradyi (Norman): III? IV? V: Samples 5, 8, 9, 11, 12 , $13,15,19,23,25,27,37,47,59,63,65,68$.

Cibicides pseudoungerianus (Cushman): III, IV, V: Samples 9, 12, 25, $27,29,39,40,42,44,45,47,53,55,59,65,68,75$.

Cibicides wuellerstorfi (Schwager): III?, V: Samples 1, 5, 7, 9, 13, 17, $23,25,29,32,34,35,37,39,40,42,47,55,57,59,61,63,70,71$, 75.

Chilostomella oolina Schwager: III, V: Sample 35.

Epistominella exigua (Brady): III, V: Samples 1, 3, 5, 8, 12, 13, 15, 17 , $21,23,25,29,34,35,40,42,45,47,49,51,55,57,63,65,67,68$ $71,73,75,77,80$

Eponides pusillus Parr: III, V: Samples 13, 19, 35, 45, 49, 59, 63, 70.
Sphaeroidina bulloides d'Orbigny: III, IV, V: Sample 1, 5, 8, 9, 17, 19 , $23,25,27,30,34,40,44,45,49,53,57,59,65,73,75$.

Trifarina bradyi Cushman: III, V: Samples 5, 12, 23, 25, 27, 47.

Bolivina difformis Williamson: IV, V: Sample 3.

Bulimina aculeata d'Orbigny: IV?, V: Sample 44.

Buliminella basicostata Parr: IV: Samples 7, 13, 15.

Cassidulina carinata Silvestri: IV, V: Sample 35.

Eggerella bradyi (Cushman): IV?, V: Samples 17, 23, 25, 29, 30, 32, $34,35,37,40,45,49,59,68,71,73,75,79,80$.

Ehrenbergina undulata Parker: IV, V: Samples 13, 15, 27, 39, 42, 45, 57.

Euuvigerina peregrina (Cushman): IV, V: Samples 7, 9, 25, 27, 29, 30, $32,34,37,71,77,79,80$.

Fissurina earlandi Parr: IV? V: Samples 21, 23.

Fissurina fissicarinata Parr f.A: IV? V: Sample 27.

Fissurina lacunata (Burrows and Holland): IV, V: Sample 5.

Fissurina semimarginata (Reuss): IV, V?: Samples 1, 9, 13, 15, 23, 25, 27.

Fissurina subrevertens Parr: IV? V: Sample 12.

Gavelinopsis praegeri (Heron-Allen and Earland): IV, V: Samples 23, $34,68$.

"Hoeglundina elegans" (d'Orbigny): IV, V: Samples 1, 15, 17, 21, 35.

Lagena costata (Williamson): IV, V: Sample 63.

Lagena distoma Parker, Jones, and Brady: IV? V: Samples 45, 51.

Lagena gracilis (Williamson): IV, V: Samples 1, 3, 9, 27, 32, 45, 73, 77.

Lagena hispidula Cushman: IV, V: Samples 8, 23.

Lagena laevis (Montagu): IV, V: Samples 13, 77.

Lagena meridionalis Wiesner: IV, V?: Samples 5, 29.

Lagena pliocenica timmsana Cushman and Gray: IV, V: Sample 5. Lagena semilineata Wright: IV, V?: Sample 80.

Lagena setosa Earland: IV, V: Sample 8.

Lagena stelligera Brady: IV, V: Samples 1, 3, 11, 30, 32, 51, 75.

Lagena striata (d'Orbigny): IV, V: Samples 30, 75, 80.

Lagena substriata Williamson: IV, V: Sample 35.

Lagena sulcata (Walker and Jacob): IV, V?: Sample 21.

Lagena sulcata var. laevicostata Cushman and Gray: IV, V: Samples $49,51$.

Lagenonodosaria scalaris (Batsch): IV, V: Sample 21.

Melonis germanicum (Ehrenberg): IV, V: Samples 1, 5, 9, 17, 68, 80.

Nummoloculina contraria (d'Orbigny): IV, V: Samples 15, 21, 23, 39, $40,44,65,80$.

Oolina globosa (Montagu): IV, V: Samples 7, 25, 35, 40, 57, 61, 63, 68, $71,73$.

Oolina hexagona (Williamson): IV, V: Samples 12, 15, 34, 80.

Oolina melo d'Orbigny: IV, V: Samples 7, 61.

Oridorsalis umbonatus (Reuss): IV? V: Samples 1, 19, 21, 29, 35, 37, $39,42,44,47,53,57,59,61,67,68,70,71,73,75,77,79,80$.

Oridorsalis umbonatus tener (Brady): IV, V: Samples 8, 17, 35, 37, 40, 63.

Parafissurina fusuliformis Loeblich and Tappan: IV, V?: Sample 5.

Parafissurina lata Parr: IV, V: Sample 27.

Parafissurina marginata Parr: IV, V: Samples 17, 21.

Parafissurina ovata Parr: IV, V?: Samples 37, 73.

Parafissurina quadrata Parr: IV, V: Sample 9.

Parafissurina subcarinata Parr: IV, V: Samples 3, 65.

Parafissurina tectulostoma Loeblich and Tappan: IV, V?: Samples 21, $39,53,59,71,80$.

"Sigmoilina" tenuis (Czjzek): IV, V: Samples 13, 15, 39, 42, 67, 79.

Uvigerina parvula Cushman: IV, V: Sample 32.

Virgulina bradyi Cushman: IV, V: Sample 37.

"Virgulina" complanata Earland: IV, V: Sample 71.

"Virgulina" spinosa Heron-Allen and Earland: IV, V: Sample 1.

"Virgulina" subdepressa Brady: IV?, V: Sample 75.

Ammobaculites agglutinans d'Orbigny var. filiformis Earland: V: Sample 44.

Astronohion tumidum Cushman and Edwards: V: Samples 13, 17, 23, $27,47$.

Bolivina decussata Brady: V: Samples 9, 15.

Bolivina globulosa Cushman: V: Sample 29.

Bolivina oceanica Cushman: V: Samples 1, 17, 30, 45, 47, 49, 51, 53, $55,57$.

Bolivina subaenariensis Cushman var.: V: Samples 3, 37, 49, 55, 57, 59 , $63,70$.

Cassidulina delicata Cushman: V: Samples 1, 9, 11, 12, 17, 23, 29, 35, $40,55,67$.

Cibicides bradyi (Trauth): V: Samples 7, 8, 9, 12, 13, 15, 21, 23, 29, 37, $40,44,47,49,55$.

Cibicides bradii Tolmachoff: V: Samples 3, 7, 12, 15, 19, 29, 30, 32, 51. 
Cibicides cicatricosus (Schwager): V: Samples 7, 12, 23, 25, 27, 30, 34, $39,79$.

Cibicides robertsonianus Brady: V: Samples 34, 51, 59, 61, 70, 79. Cibicides subhaidingerii Parr: V: Sample 79.

Dentalina advena (Cushman): V: Samples 42, 68.

Dentalina filiformis (d'Orbigny): V: Sample 9.

Dentalina subemaciata Parr: V: Samples 61, 77.

Ehrenbergina trigona (Goës): V: 34, 37.

Epistominella rugosa convexa Parker: V: Sample 35.

Epistominella umbonifer (Cushman): V: Samples 73, 75.

Eponides bradyi Earland: V: Samples 61, 80.

Eponides tumidulus (Brady): V: Samples 29, 39, 71, 73.

Fissurina alveolata (Brady): V: Samples 40, 70.

Fissurina auriculata (Brady): V: Samples 9, 12, 17, 29, 34, 47, 63, 65.

Fissurina auriculata duplicata Sidebottom: V: Sample 39.

Fissurina exsculpta (Brady): V: Sample 12.

Fissurina lagenoides (Williamson): V: Sample 5.

Fissurina longispina (Brady): V: Sample 8.

Fissurina marginata (Walker and Boys): V: Samples 12, 13, 15, 19, 27, $29,35,42,44,45,63,68,77,79,80$.

Fissurina radiata Seguenza: V: Sample 21.

Gavelinopsis lobatulus (Parr): V: Samples 1, 3, 9, 13, 19, 21, 29, 67.

Gyroidina acuta Boomgaart: V: Samples 27, 34, 39, 67.

Gyroidina broeckhiana (Karrer): V: Samples 7, 25, 29, 34.

Gyroidina delicata Parker: V: Samples 5, 55, 61, 65.

Gyroidina neosoldanii Brotzen: V: Samples 5, 17, 29, 34, 40, 42, 44, 53, $63,68,70,71$.

Gyroidina nitidula Schwager: V: Samples 3, 5, 7, 12, 21, 25, 29, 37, 44, $49,73,75,77,79,80$.

Gyroidina quinqueloba Uchio: V: Sample 34

Gyroidina umbonata Silvestri: V: Samples 19, 23.

Karreriella bradyi (Cushman): V: Samples 1, 7, 9, 13, 15, 17, 19, 23, $27,30,34,39,45,55,59$.

Karreriella novangliae (Cushman): V: Sample 35.

Lagena bicarinata Sidebottom var. spinigera Parr: V: Sample 12.

Lagena hispida Reuss: V: Samples 15, 35.

Lagena lyelli (Seguenza): V: Samples 5, 27, 34.

Lagena nebulosa Cushman: V: Sample 68.

Lagena sidebottomi Earland: V: Sample 5.

Lagena staphyllearia Schwager: V: Samples 1, 67.

Laticarinina pauperata (Stache): V: Samples 7, 9, 12, 19, 21, 27, 29, 30, $32,37,40,42,45,49,61,68,70,79,80$.

Martinottiella communis (d'Orbigny): V: Sample 44.

Melonis pompilioides (Fichtel and Moll): V: Samples 13, 35, 71, 73.

Nodosaria albatrossi Cushman: V: Samples 45, 47, 51, 68.

Nodosaria doliolaris Parr: V: Samples 59, 67.

Nodosaria vertebralis (Batsch): V: Sample 53.
Nummoloculina ? irregularis (d'Orbigny): V: Sample 79.

Oolina felsinea (Fornasini): V: Samples 5, 7, 9, 13, 15, 21, 23, 27, 34, $35,67$.

Orthomorphina challengerina Thalmann: V: Samples 1, 12, 25, 34, 63.

Osangularia bengalensis (Schwager): V: Samples 12, 37, 39, 40, 42, 59, 67.

Parafissurina quadrata (Williamson) var. carinata (Chapman): V: Sample 15.

Planodiscorbis circularis Sidebottom: V: Sample 39.

Planulina ariminensis d'Orbigny: V: Samples 3, 29.

Pleurostomella acuminata Cushman: V: Samples 1, 5, 12, 13, 15, 17, $19,21,23,25,27,34,55,59,63,67,68,75,77,80$.

Pleurostomella alternans Schwager: V: Samples 1, 21, 47, 49, 55, 57, $61,73$.

Pleurostomella brevis Schwager: V: Samples 15, 25, 45, 49, 51, 55, 65, 67.

Pleurostomella rapa recens (Dervieux): V: Sample 19.

Pleurostomellina sp. nov.: V: Samples 3, 7, 15, 19, 40, 44, 49, 59, 63, $67,70,73,75,77$.

Pyrgo murrhyna (Schwager): V: Samples 3, 21, 35, 71.

Pyrulina angusta (Egger): V: Samples 7, 19, 21, 25, 27, 34, 39, 42, 77.

Pullenia bulloides (d'Orbigny): V: Samples 7, 12, 17, 19, 21, 23, 25, 32, $35,37,39,40,44,45,47,49,51,53,55,57,59,61,63,67,68,70$, $71,75,77,79,80$.

Pullenia quadriloba (Reuss): V: Sample 59.

Pullenia quinqueloba (Reuss): V: Samples 1, 3, 7, 13, 15, 19, 21, 25, 27, $34,45,47,49,53,57,61,71,73,80$.

Quinqueloculina venusta Karrer: V: Samples 19, 21.

Recloglandulina aequalis (Reuss): V: Sample 77.

Sigmoilopsis schlumbergeri (Silvestri): V: Samples 3, 8, 9, 11, 13, 15, $19,23,29,35,37,53,55$.

Siphouvigerina proboscidea (Schwager): V: Samples 5, 11, 15, 17, 21, $25,27,29,53,71$.

Spirophthalmidium pusillum (Earland): V: Samples 12, 17, 35, 47, 59, $71,73$.

Stilostomella antillea (Cushmam): V: Samples 1, 3, 9, 11, 12, 13, 15, 17, $19,21,23,25,27,29,30,34,37,39,42,44,49,53,55,57,59,61$, $63,65,73,79,80$.

Stilostomella bradyi (Cushman): V: Samples 9, 21.

Stilostomella consobrina (d'Orbigny): V: Samples 1, 3, 5, 7, 9, 11, 12, $13,15,17,19,21,23,25,27,29,30,34,37,40,42,44,45,47,53$, $59,63,70,71,73,75,79,80$.

Uvigerina asperula Czjzek: V: Samples 8, 39, 42, 65, 67, 68.

Uvigerina auberiana d'Orbigny var. laevis Goës: V: Samples 9, 34, 65.

Uvigerina auberiana d'Orbigny f. typica Boltovskoy: V: Sample 9.

Uvigerina hollicki Thalmann: V: Samples 7, 12, 37, 70. 